\title{
CAMBIO CLIMÁTICO EN TIEMPOS DE EMERGENCIA. LAS COMUNIDADES AUTÓNOMAS EN LAS VEREDAS DEL "FEDERALISMO CLIMÁTICO" ESPAÑOL ${ }^{1}$
}

\author{
CLIMATE CHANGE IN EMERGENCY TIMES. THE AUTONOMOUS \\ COMMUNITIES IN THE PATHS OF THE SPANISH "CLIMATIC \\ FEDERALISM" \\ ENDRIUS COCCIOLO \\ Departament de Dret Públic - Centre d'Estudis de Dret Ambiental (CEDAT) \\ Universitat Rovira i Virgili \\ endrius.cocciolo@urv.cat
}

\begin{abstract}
"Ante el problema del cambio climático, vuelve a reconocerse que las nuevas soluciones sólo se hallarán implicando a los poderes locales y [sub-]estatales [...] si se aprobara una legislación rígida centralizada no cabría innovar a través de experimentos parciales. Siempre habrá esta válvula de escape para que en el ámbito estatal, regional o local se pueda experimentar con regulaciones más innovadoras y así, posteriormente, tener una implantación global".

Manuel Ballbé, "El futuro del Derecho administrativo en la globalización: entre la americanización y la europeización", Revista de Administración Pública, núm. 174, 2007, p. 259
\end{abstract}

Sumario: 1. El contexto: la pandemia de la COVID-19 y la emergencia climática; 2. Emergencia climática, emergencia constitucional y derecho del cambio climático.

\footnotetext{
${ }^{1}$ Este trabajo ha sido desarrollado en el marco del proyecto de investigación Constitución climática global: gobernanza y Derecho en un contexto complejo, financiado por el Ministerio de Economía y Competitividad para el trienio 2017-2019 (proyecto DER2016-80011-P; investigadores principales: Jordi Jaria-Manzano y Susana Borràs Pentinat).
} 


\section{El contexto: la pandemia de la COVID-19 y la emergencia climática}

Este número monográfico de la Revista Catalana de Dret Ambiental - dedicado al estudio de la normativa autonómica y de la estatal venidera dirigida a abordar los retos del cambio climáticos y de la transición energética - aparece en un momento histórico especialmente difícil y complejo. La crisis sanitaria global causada por la pandemia de la COVID-19 ya registra más de 10.000.000 de casos confirmados y más de 500.000 muertes, mientras continúa avanzado su difusión, según los datos de la Organización Mundial de la Salud ${ }^{2}$. Esta crisis sanitaria tiene lugar en tiempos de una emergencia climática cuyos efectos serán aún más sobrecogedores que aquellos derivados de la pandemia, si no se toman medidas estrictas y coherentes durante esta segunda década del siglo XXI para evitar un colapso ambiental catastrófico que, como han alertado los científicos del Panel Intergubernamental del Cambio Climático (IPCC), daría lugar a situaciones de extrema pobreza para cientos de millones de personas y al consiguiente aumento de las desigualdades planetarias ${ }^{3}$.

El cambio climático y la pérdida de biodiversidad representan los llamados "límites principales" (core boundaries) en el marco de los nueve límites planetarios (Planetary Boundaries Framework, PBF) que delimitan un "espacio operativo seguro" para la humanidad basado en los procesos biofísicos intrínsecos que regulan la estabilidad del sistema terrestre ${ }^{4}$. Los nueve parámetros considerados en el PBF incluyen las interacciones de suelo, océano,

\footnotetext{
2 Información recabada de la página oficial de la World Health Organization, WHO Coronavirus Disease (COVID-19) Dashboard: https://covid19. who.int (fecha de última consulta: 27/06/2020).

${ }^{3}$ Los científicos del IPCC han advertido, en el Global Warming of $1,5^{\circ} \mathrm{C}$, Special Report de 2018 , que sólo solo hay tiempo hasta el 2030 para tener una posibilidad razonable de limitar el calentamiento global a $1,5^{\circ} \mathrm{C}$. Alcanzar este objetivo en un decenio "would require rapid and farreaching transitions in energy, land, urban and infrastructure (including transport and buildings), and industrial systems [...]. These systems transitions are unprecedented in terms of scale, [...] and imply deep emissions reductions in all sectors [...]". Las implicaciones de esta advertencia son claras: el objetivo establecido en el Acuerdo de París de mantener el aumento de la temperatura media mundial muy por debajo de $2^{\circ} \mathrm{C}$ respeto a los niveles preindustriales, debe considerarse insuficiente, mientras que el objetivo más ambicioso de limitar el aumento de la temperatura a $1,5^{\circ} \mathrm{C}$ se convierte en la verdadera meta (difícil) a alcanzar. Sin embargo, el informe también afirma que el objetivo de detener el calentamiento global a $1,5^{\circ} \mathrm{C}$ podría ser todavía técnicamente factible, en particular si hay una respuesta firme e inmediata de los gobiernos. Para más informaciones, véase: IPCC, Global Warming of $1,5^{\circ} \mathrm{C}$, Special Report, 2018, Headline Statements.

${ }^{4}$ Johan Rockström et al. "Planetary boundaries: Exploring the safe operating space for humanity", Ecology and Society, Vol. 14, no. 2, art. 32, 2009.
} 
atmósfera y formas de vida que, en su conjunto, crean las condiciones de las cuales depende la subsistencia de la humanidad. Cuatro de estos nueve límites planetarios se han cruzado como resultado de la actividad humana: el cambio climático, la pérdida de integridad de la biosfera, el cambio el sistema del suelo y la alteración de los ciclos biogeoquímicos del fósforo y nitrógeno ${ }^{5}$. La comprensión cada vez mayor de los impactos e interdependencias de las sociedades humanas con los procesos biogeoquímicos esenciales para sostener la vida en la Tierra condujo a la identificación de una nueva época geológica del planeta que se denomina Antropoceno ${ }^{6}$, cuyo comienzo ha sido establecido en coincidencia con los inicios de era nuclear y de la "Gran Acceleración" después de 1945, según la votación vinculante del panel de expertos que integran el Anthropocene Working Group (AWG) y que conducirá a una decisión final sobre la nueva era geológica por la International Commission on Stratigraphy (ICS) ${ }^{8}$. De esta transición ecológica derivan múltiples consecuencias para el Derecho y la justicia9. En cualquier caso, "por lo que sabemos, el Antropoceno podría ser

${ }^{5}$ Ibid.

${ }^{6}$ Paul J. Crutzen, Eugene F. Stoermer, "The 'Anthropoceneo"', Global Change Newsletter, 41, 2000, pp. 17-18; Crutzen, "Geology of mankind", Nature. Nature Publishing Group, 415(6867), 2002, pp. 23-23.

${ }^{7}$ Según los científicos, en cuanto a la determinación de los inicios del Antropoceno, es posible concluir lo siguiente: "Its beginning would be optimally placed in the mid-20th century, coinciding with the array of geological proxy signals preserved within recently accumulated strata and resulting from the 'Great Acceleration' of population growth, industrialization and globalization; The sharpest and most globally synchronous of these signals, that may form a primary marker, is made by the artificial radionuclides spread worldwide by the thermonuclear bomb tests from the early 1950s". Véase: "Working Group on the 'Anthropocene' (AWG), Results of binding vote by AWG Released 21st May 2019, http://quaternary.stratigraphy.org/working-groups/anthropocene/ (fecha de última consulta: 27/06/2020)

${ }^{8}$ Meera Subramanian, "Anthropocene now: influential panel votes to recognize Earth's new epoch", Nature, News, 21 May 2019, https://www.nature.com/articles/d41586-019-01641-5 (fecha de última consulta 27/06/2020)

${ }^{9}$ Sobre esta cuestión, entre otros, véase: Louis Kotzé, Rakhyun E. Kim, "Earth System Law: The Juridical Dimensions of Earth System Governance”, Earth System Governance, Vol. 1, 2019, pp. 1-12; Louis Kotzé, "Earth System Law for the Anthropocene", Sustainability, 11(23), 2019, p. 6796; Louis Kotzé, "Earth system law for the Anthropocene: rethinking environmental law alongside the Earth system metaphor", Transnational Legal Theory, Vol. 11, Issue 1-2, 2020, pp. 75-104; Antonio Cardesa-Salzmann, Endrius Cocciolo, "Global Governance, Sustainability and the Earth System: Critical Reflections on the Role of Global Law', Transnational Environmental Law, Cambridge University Press, 2019, pp. 1-25 ; Endrius Cocciolo,"Capitalocene, Thermocene and the Earth System: Global Law and Connectivity in the Anthropocene Age", en Jaria-Manzano, J. and Borràs, S. (eds) Research Handbook on Global Climate Constitutionalism. Cheltenham: Edward Elgar, 2019, pp. 277-301; Frank Biermann, Agni Kalfagiann, "Planetary justice: A research framework", Earth System Governance, 2020, pp. 1-11; Jordi Jaria-Manzano, "Law in the Anthropocene", Jaria-Manzano, J. and Borràs, S. (eds), Research Handbook on Global Climate Constitutionalism. Cheltenham: Edward Elgar, 2019, pp. 31-49; Jordi Jaria-Manzano, La 
una época rica en amenazas existenciales"10. En este sentido, los estudios ponen de manifiesto cómo las autoridades públicas y los sistemas de gobernanza "han fallado en abordar las formas en que los recientes cambios en la economía política internacional se combinan con las inestabilidades ambientales del Antropoceno, provocando el aumento de los riesgos epidemiológicos"11. Asimismo, se han evidenciado las relaciones de causalidad entre contaminantes de origen antrópico, cambio climático y el aumento de eventos pandémicos o epidémicos ${ }^{12}$.

Sin embargo, como han evidenciado Manzanedo y Manning, la crisis de la COVID-19 nos brinda una visión sin precedentes sobre cómo gestionar la crisis climática global, debido a que hay muchos paralelismos entre la primera y la segunda ${ }^{13}$. En este sentido las lecciones más importantes a tener presentes son las siguientes: 1) Las graves consecuencias resultantes de ignorar los Ilamamientos científicos para actuar de forma temprana y preventiva, aunque las medidas reclamadas parezcan radicales; 2) Entender que sobrepasar ciertos umbrales provoca cambios irreversibles que reducen de forma significativa la capacidad de control de procesos con desarrollos difícilmente previsibles; 3) El cambio climático y la pandemia no tienen el mismo impacto social, pues los colectivos más vulnerables resultan mayormente afectados por estas emergencias $y$, por lo tanto, es necesario prever las medidas de protección necesarias para minimizar las injusticias resultantes; 4) La debilitación de la solidaridad internacional experimentada durante la crisis de la COVID-19 puede alcanzar niveles aún mayores en los eventos derivados del cambio climático, por ende, es preciso diseñar mecanismos sólidos de intervención y cooperación; 5)

Constitución del Antropoceno, Valencia: Tirant lo Blanch, 2020.

${ }^{10}$ Mario Herrero, Philip Thornton, "What can COVID-19 teach us about responding to climate change?", The Lancet Planetary Health, Volume 4, Issue 5, May 2020, p. e174 (traducción propia)

${ }^{11}$ Katherine Hirschfeld, "Microbial insurgency: Theorizing global health in the Anthropocene", The Anthropocene Review, Volume 7, Issue 1, 2020, pp. 3-18 (traducción propia).

${ }^{12}$ Aristidis Tsatsakis et al., "COVID-19, an opportunity to reevaluate the correlation between longterm effects of anthropogenic pollutants on viral epidemic/pandemic events and prevalence", Food and Chemical Toxicology, Volume 141, July 2020, 111418;

13 Rubén D. Manzanedo, Peter Manning, "COVID-19: Lessons for the climate change emergency", Science of the Total Environment, 2020 (disponible on line desde el 27 de junio de 2020: https://www.sciencedirect.com/science/article/pii/S0048969720340857) 
Los gastos para prevenir riesgos resultan ser inversiones más eficientes que los costes por los daños que no se han abordado de forma precautoria.

Por de pronto, de las respuestas institucionales a la crisis sanitaria global hemos observado que acciones masivas, rápidas y sin precedentes son posibles en poco tiempo, por lo tanto, debería ser también posible movilizar en la década 2020-2030 los recursos necesarios para hacer frente a los retos de la mitigación y de la adaptación al cambio climático, tal como señalan Herrero y Thornton:

[G]overnments have found \$8 trillion to help combat the spread and effects of COVID-19 in just 10 weeks. Surely, governments can find $\$ 1 \cdot 8$ trillion in the coming decade to combat the effects of climate change on food and livelihood systems [...], while at the same time pursuing aggressive emission reductions to achieve the $1.5^{\circ} \mathrm{C}$ global warming goal. We should treat this situation with the same urgency as the COVID-19 pandemic, before we risk additional disruptions of incalculable magnitudes. ${ }^{14}$

\section{Emergencia climática, emergencia constitucional y derecho del cambio climático}

A partir de la reformulación del argumento de Kjaer sobre las condiciones que dan lugar a una crisis global, la actual emergencia resultante de la combinación de la emergencia climática, de la pandemia y de la crisis económica derivada de la crisis sanitaria, puede entenderse como la manifestación de un desajuste crítico entre el Sistema Tierra, la composición estructural de la sociedad mundial y las arquitecturas constitucionales existentes ${ }^{15}$. Como resultado de este desequilibrio, la reproducción social provoca una aceleración y profundización de la actual crisis socio-ecológica y de las injusticias a escala planetaria.

Hasta la fecha en la que se redactan estas líneas, 1.736 entre gobiernos y otras instituciones públicas de 30 Países, que abarcan 820 millones de ciudadanos, han declarado la emergencia climática ${ }^{16}$. Entre ellas cabe señalar:

\footnotetext{
${ }^{14}$ Herrero, Thornton, "What can COVID-19 teach us...", cit.

${ }^{15}$ Poul Kjaer, "Law and Order Within and Beyond National Configuration". Poul Kjaer, Gunther Teubner, Alberto Frebbrajo (eds.) The Financial Crisis in Constitutional Perspective: The Dark Side of Functinal Differentiation, Hart, 2011, p. 395.
}

16 Para más información, véase la Declaración de Emergencia Climática: https://climateemergencydeclaration.org/climate-emergency-declarations-cover-15-millioncitizens/ (fecha de última consulta: 28/06/2020). 
- La Declaración de emergencia climática y ambiental adoptada por el Parlamento Europeo el 28 de noviembre de 2019, en vísperas de la COP25, pidiendo "a la Comisión, a los Estados miembros y a todos los actores mundiales, que adopten urgentemente las medidas concretas necesarias para luchar y contener esta amenaza antes de que sea demasiado tarde"17.

- La Declaración de emergencia climática y ambiental del Gobierno de España, adoptada por Acuerdo del Consejo de Ministros del 21 de enero de 2020, por la que el ejecutivo se comprometió a una serie de medidas entre las cuales estaba la remisión al Parlamento del proyecto de Ley de Cambio Climático.

- En consideración de las leyes autonómicas ya vigentes, debe hacerse mención también a las declaraciones de emergencia climática del Gobierno de la Generalitat de Catalunya del 14 de mayo de 2019 y del Gobierno de las Islas Baleares del 8 de noviembre de 2019, mientras que en Andalucía - a pesar de que en octubre de 2018 fue aprobada una Ley de medidas frente al cambio climático - el pasado 6 de febrero de 2020, los partidos que integran la mayoría de gobierno rechazaron en el Parlamento instar a la Junta a declarar la emergencia climática.

Detrás de estas enfáticas declaraciones políticas (carecen, pues, de cualquier efecto jurídico vinculante) se asoma, en palabras de Carducci, una verdadera emergencia constitucional ${ }^{18}$ :

"Siamo di fronte a una emersione espressiva di un sistema complesso di interazioni caotiche, catene causali, responsabilità diffuse, specifiche e plurime, su cui il diritto si dovrebbe interrogare con forza; e lo dovrebbe fare soprattutto il diritto costituzionale, evolutosi nella moltiplicazione di bisogni materiali attraverso regole di manipolazione dei cicli energetici della natura (nell'epoca contemporanea dei due ultimi secoli e mezzo dell'energia fossile)"19.

\footnotetext{
17 Resolución del Parlamento Europeo, de 28 de noviembre de 2019, sobre la situación de emergencia climática y medioambiental [2019/2930(RSP)].

${ }^{18}$ Michele Carducci, II diritto costituzionale "durante" la catástrofe climática. laCostituzione.info, 21 Sepetember 2019, disponible en: https://www.lacostituzione.info/index.php/2019/09/21/ildiritto-costituzionale-durante-la-catastrofe-climatical (fecha de última consulta: 23/06/2020).

${ }^{19} \mathrm{lbid}$
} 
El citado autor, subraya el carácter ecológicamente disfuncional del Derecho de nuestro tiempo ${ }^{20}$. En efecto, la alteración de la naturaleza por las acciones antrópicas está arraigada en los propios orígenes de las instituciones jurídicas, lo que explica las contradicciones con las normativas destinadas a proteger el medio ambiente. No puede olvidarse que la constitucionalización de los sistemas jurídicos nacionales tiene sus raíces en la revolución industrial basada en el carbón. En este marco, el derecho ambiental ha surgido como correctivo para las externalidades negativas y las ineficiencias ecológicas de un desarrollo social sustentado en un "derecho constitucional fósil"21 al que corresponde, en palabras de Mitchell, un modelo históricamente consolidado de "carbon democracy"22. Por estos motivos cuando se plamtean reformas destinadas a la transición energética, para que ésta tenga sentido y no quede reducida a una mera cuestión tecnológica y gerencial, deben proyectarse como un conjunto de medidas orientadas a lograr nuevas formas de democracia energética ${ }^{23}$.

Este "derecho constitucional fósil" tiene dos vertientes: una global y otra nacional. En efecto, cuando se habla de la progresiva constitucionalización del espacio jurídico global, y muy especialmente, del constitucionalismo ambiental ${ }^{24}$ y climático ${ }^{25}$, debe observarse que, a pesar de que los combustibles fósiles están en el núcleo de la cuestión climática, en el régimen climático global no se menciona la cuestión energética que queda "estratégicamente declimatizada"26

\footnotetext{
${ }^{20}$ Michele Carducci, 'Demodiversità" e Futuro Ecologico', in Bagni, S. (ed.), Come Governare L'Ecosistema? Un Approccio Multidisciplinare, Bologna: Dipartimento di Scienze Giuridiche, Università di Bologna, 2018, p. 65.

${ }^{21}$ Carducci, '"Demodiversità" e Futuro Ecologico', cit.

22 Timoty Mitchell, Carbon Democracy. Political Power in the Age of Oil. Verso, 2011.

${ }^{23}$ Sobre este tema, véase: Endrius Cocciolo, "Estado garante, energia y transición justa. (Re)formulación teòrica y despligue practico". M. Darnaculleta Gardella, J. Esteve Pardo, M. Ibler (coords.), Nuevos retos del Estado garante en el sector energético", Marcial Pons, 2020, pp. 211243

24 Louis Kotzé, "Arguing Global Environmental Constitutionalism", Transnational Environmental Law, Vol. 1, Issue 1, 2012, pp. 199-233; Louis Kotzé, "A Global Environmental Constitution for the Anthropocene?", Transnational Environmental Law, 2019, Vol. 8, Issue 1, pp. 11-33.

25 Jordi Jaria-Manzano, Susana Borràs (eds), Research Handbook on Global Climate Constitutionalism, Cheltenham: Edward Elgar, 2019.

${ }^{26}$ Stefan C. Aykut, Monica Castro, "The end of fossil fuels? Understanding the partial climatisation of energy policy", en Stefan C. Aykut, Jean Foyer, Edouard Morena (eds.), Globalising the Climate. COP21 and the climatisation of global debates, Routledge, 2019.
} 
en el marco de la gobernanza sectorial de la energía; de este modo se consigue mantener la cuestión energética fuera de las negociaciones climáticas y amparada en el derecho constitucional global de mercado funcionalmente diferenciado y de base fósil27, aquello que Cutler denomina "neoliberal constitutionalism" o "free market constitutionalism"28.

En la vertiente nacional, el caso de España es muy ilustrativo del arraigo del derecho constitucional fósil. En este sentido deben señalarse una serie de aspectos problemáticos: en primer lugar, los años comprendidos entre el 2012 y junio de 2018, se han caracterizado como una etapa en el que se implantó un verdadero "régimen de resistencia" a las energías renovables ${ }^{29}$, un corpus jurídico desfasado respecto a la necesaria la incorporación en nuestro ordenamiento jurídico de las estrategias e instrumentos elaborados a nivel internacional y europeo para renovar el sistema energético, de inmovilismo normativo climático y de recentralización de las competencias también en materia ambiental ${ }^{30}$. En segundo lugar, deben señalarse las críticas de Galera Rodrigo, tanto en lo que atañe a la "patrimonialización de la competencia" y la "cultura de la unilateralidad" en materia de energía ${ }^{31}$ que ha practicado el Estado, como por el el "anacronismo normativo" derivado de la propia interpretación de la Constitución Española (CE) por parte del Tribunal Constitucional (TC) $)^{32}$. En

${ }^{27}$ Cocciolo, "Capitalocene, Thermocene and the Earth System...", cit., p. 292.

${ }^{28}$ A. Claire Cutler, "The Judicialization of Private Transnational Power and Authority", Indiana Journal of Global Legal Studies, vol. 25, Issue 1, 2008, p. 64.

29 Iñigo Capellán-Pérez, Àlvaro Campos-Celador, Jon Terés-Zubiaga, "Renewable Energy Cooperatives as an instrument towards the energy transition in Spain", Energy Policy, 2018, 123, pp. 215-229; Jose Francisco Alenza García, "Las energías renovables ante la fugacidad legislativa: la mitificación de los principios de (in)seguridad juridical y de (des)confianza legítima: a propósito de la STC 270/2015 sobre el nuevo sistema retributivo de las energias renovables" en Actualidad Jurídica Ambiental, 2016, núm. 55, pp. 1-19

${ }^{30}$ Lucia Casado Casado, La recentralización de competencias en material de protección del medio ambiente, Generalitat de Catalunya. Institut d'Estudis de l'Autogovern. 2018, Barcelona; Alba Nogueira López, "El demediado derecho a un medio ambiente adecuado" en benigno Pendás (Dir.), Esther González, Rafael Rubio, (Coords.) España Constitucional (1978-2018) Trayectorias y perspectivas III, 2018, pp. 2465-2482; Alba Nogueira López (2017): Pack Premium o pack pack básico. ¿Ordenamiento dual? en Revista Aranzadi de Derecho Ambiental, núm 38, 2017, pp. 297-325.

${ }^{31}$ Galera Rodrigo, Susana (2018): "Transición energética: un salto cualitativo en el progreso democrático", Ambienta, 2018, 125, p. 83.

32 Según Galera Rodrigo: "[R]esulta necesario recordar que el entendimiento en la segunda década del siglo XXI de lo que ha de entenderse por «energía» no puede ser idéntico al que en 1978 subyacía en el mismo término cuando el legislador constitucional lo plasma en el artículo 149.1.25: puede ser cuestionable asociar sin más todas las modalidades de energías renovables 
efecto no se entiende que, rozando la tercera década del siglo XXI, el TC siga mirando a la energía de la misma manera que los constituyentes de 1978. En primer lugar, porque no está claro que el título competencial que en la Constitución amparaba esencialmente los hidrocarburos y la energía nuclear tenga hoy en día que identificar al mismo titular de la competencia cuando nos refiramos a las energías renovables en un sistema cada vez más descentralizado y de generación distribuida ${ }^{33}$. En segundo lugar, porque debería considerarse superada la interpretación que vincula preferentemente el título "Energía" con el título sobre bases y coordinación de la actividad económica (art. 149.1.13 CE), como resultado de la "ambientalización" de la energía en clave europea"34, por lo que el título "Energía" debería leerse en estricta conexión con el 149.1.23. Y finalmente, porque, a falta de un título específico sobre cambio climático en la CE, el art. 149.1.25 debe necesariamente "climatizarse", ya que las políticas de lucha contra el cambio climático implican necesariamente medidas energéticas. Con este telón de fondo, solo desde el último trimestre de 2018 en España se ha empezado a dar pasos, algunos tímidos y otros más atrevidos - especialmente por parte de ciertas comunidades autónomas y entes locales - en una dirección más propicia para abordar los retos del cambio climático y la transición energética. Así, ante la "inactividad climática" del gobierno central, inicialmente fue en Catalunya donde se aprobó la pionera Ley 16/2017, de 1 de agosto, del cambio climático, seguida de la Ley $8 / 2018$, de 8 de octubre, de medidas frente al cambio climático y para la transición hacia un nuevo modelo energético en Andalucía, a la que se sumó la tercera ley autonómica, la Ley 10/2019, de 22 de febrero, de Transición Energética y Cambio Climático de las Islas Baleares. De esta manera, en el Estado autonómico - que algunos han llegado a calificar, con cierto atrevimiento, como el más descentralizado de Europa - parecía vislumbrase un incipiente camino hacia un "federalismo climático". Sin embargo, la sentencia del Tribunal Constitucional 87/2019, de 20 de junio, sobre la Ley

con los hidrocarburos; es ya inconcebible que el título 'energía' mantenga su insistente asociación con la 'coordinación de la economía' mientras permanece ajeno al proceso de ambientalización que se ha operado en el ámbito europeo; y resulta una fuente de conflictos mantener la literalidad, y sobre todo la interpretación, de estos preceptos en la Constitución mientras los Estatutos de Autonomía reflejan la siguiente 'generación', por así decirlo, a los que han dado lugar.", ibid. P. 84-85.

33 Ibid.

34 Ibid. 
catalana 16/2017 del Cambio Climático, ha dejado aquel camino en una vereda y ha vuelto a utilizar como parámetro de constitucionalidad la tradicional interpretación fósil, economicista y centralista del título competencial en materia de energía. En efecto, en esta sentencia no llama la atención la previsibilidad de su fallo, sino su desoladora y trasnochada argumentación jurídica. Para usar las palabras de Alenza García, ya estábamos acostumbrados a los "partos en los montes" del Tribunal Constitucional que, en ocasión de la STC 270/2015 sobre el RD-L 9/2013 de medidas urgentes para garantizar la estabilidad del sistema eléctrico (sustitución del régimen retributivo de las $E R$ ), había dictado con una sentencia "insustancial, gris, decepcionante en el fondo y en la forma, con una argumentación escasa, endeble y puramente formal"35. Pues bien, el TC ha resuelto el recurso de inconstitucionalidad contra la ley catalana con una decisión aún más gris y desconectada del contexto de crisis climática en el que vivimos. EI TC hubiera podido construir una argumentación a partir del art. 45.2 CE para desarrollar un discurso jurídico sólido sobre el clima. Tal como ha afirmado JariaManzano, en esta norma se establece la obligación para los poderes públicos de velar por la utilización racional de tos los recursos naturales - obligación que debe extenderse al uso de los combustibles fósiles, en la medida en que eso afecta a la composición de la atmósfera - con el fin de proteger y mejorar la calidad y de la vida y defender y restaurar el medio ambiente ${ }^{36}$. En lugar de ello, el Tribunal Constitucional ha recordado que:

"Las normas actualmente vigentes prevén, de hecho, un sistema energético [...] en el que se admite el uso de combustibles fósiles y la energía nuclear, y que no aparece sujeto a plazo. Así, el Estado reconoce expresamente el derecho de los productores de energía eléctrica a "la utilización en sus unidades de producción de aquellas fuentes de energía primaria que consideren más adecuadas" [art. 26.1 a) de la Ley 24/2013, de 26 de diciembre, del sector eléctrico, de carácter básico al amparo de lo establecido en el artículo 149.1 .13 y 25 de la Constitución según su disposición final segunda], reconoce también el derecho a la explotación y aprovechamiento de combustibles fósiles (art. 2 de la Ley 22/1973,

\footnotetext{
${ }^{35}$ Alenza García, "Las energías renovables ante la fugacidad legislativa...", cit., p. 7-8.

${ }^{36}$ Jordi Jaria-Manzano, "La litigació climática a Espanya: una prospectiva", Revista Catalana de Dret Ambiental, Vol. IX, núm. 2, 2018, pp. 21-22.
} 
de 21 de julio, de minas, y Ley 54/1980, de 5 de noviembre, de modificación de la anterior con especial atención a los recursos minerales energéticos) y admite igualmente la existencia de instalaciones nucleares (arts. 28 y ss. de la Ley $25 / 1964$, de 29 de abril, sobre energía nuclear), normas todas ellas a las que hay que reconocer forzosamente carácter materialmente básico al amparo de los núms. 13 y 25 del art. 149.1 CE”. (FJ 10)

Y para rematar el asunto, después de analizar el art. 19 (energía) de la ley catalana, cuyo objetivo era la transición energética hacia un modelo cien por cien renovable, desnuclearizado y descarbonizado, el Tribunal Constitucional ha concluido que:

“[...] no pueden las comunidades autónomas decidir libre, aislada e individualmente si, y en su caso cómo, afrontan esta "transición energética", y la fecha en que debe conseguirse ésta, a modo de dies ad quem. Solo el Estado se encuentra en la posición y tiene las herramientas para decidir y planificar esa transformación. No solo por tener encomendadas las competencias ya señaladas de los núms. 13, 23 y 25 del art. 149.1, con el contenido que resulta de la doctrina constitucional citada en el precedente fundamento jurídico 4 , sino por la necesaria coordinación con los restantes Estados miembros de la Unión Europea y con la propia Unión, con competencia en la materia, pues en un espacio sin fronteras interiores y libre circulación de mercancías, servicios personas y capitales (art. 26.2 del tratado de funcionamiento de la Unión Europea) solo actuando de manera conjunta puede afrontarse esa transformación".

Esta decisión termina siendo paradigmática: socava el camino hacia una verdadera transición energética, ignora las dimensiones y las demandas de justica energética y climática, tiene una concepción centralizada y limitada de democracia y, peor aún, ignora la urgencia del problema.

Solo en fechas muy recientes, una vez superada una fase de instabilidad política, (y aún en el medio de la pandemia) se puede decir que hemos entrado en una nueva etapa para el derecho climático español: por fin, el pasado 19 de mayo de 2020 el Consejo de Ministros ha remitido a las Cortes el primer proyecto de Ley de Cambio Climático y Transición Energética (LCCTE) para que España alcance 
la neutralidad de emisiones no más tarde de 2050 , en consonancia con los objetivos establecidos por el Green Deal europeo ${ }^{37}$. En el contexto de la crisis de la COVID19, y ante la expectativa de que la tramitación parlamentaria de la LCCTE se alargue, el Gobierno ha decidido adelantar algunos contenidos del proyecto de ley, mediante el Real Decreto-ley 23/2020, de 23 de junio, por el que se aprueban medidas en materia de energía y en otros ámbitos para la reactivación económica, considerando que:

\begin{abstract}
"En estas circunstancias excepcionales, la política energética debe estar orientada, teniendo en cuenta su objeto y ámbito de aplicación en la actual Transición Energética, a impulsar una serie de medidas que favorezcan la recuperación económica, la movilización de recursos financieros tanto nacionales como de la Unión Europea, la creación de empleo sostenible, y la necesaria colaboración entre las políticas presupuestarias, monetarias, financieras y estructurales" ${ }^{\text {" }}$.
\end{abstract}

La actual posición política del ejecutivo permite cierto optimismo respecto a las posibilidades de que las Comunidades Autónomas puedan definan estrategias más ambiciosas para afrontar el cambio climático y la transición energética.

Muchas de las cuestiones a las que nos hemos referido serán analizadas de forma detallada por los trabajos que integran la sección monográfica de este número de la Revista.

La Profesora Alba Nogueira López, lleva a cabo una lectura horizontal de las leyes autonómicas en materia de cambio climático, dentro del encuadre competencial y en consideración de la proyección de los Estatutos de Autonomía en las competencias en materia de clima y energía. El análisis pone de manifiesto, no solo las poderosas tensiones de naturaleza competencial que experimentan las Comunidades Autónomas, sino también aquellas que derivan de una interpretación en la que se priman las reglas prevalentes del modelo

\footnotetext{
${ }^{37}$ Comunicación de la Comisión, El Pacto Verde Europeo, COM(2019) 640, disponible on line: https://eur-lex.europa.eu/legal-content/ES/TXT/?uri=CELEX:52019DC0640 (fecha de último acceso: 28/06/2020).

${ }^{38}$ Exposición de motivos del Real Decreto-ley 23/2020, de 23 de junio, por el que se aprueban medidas en materia de energía y en otros ámbitos para la reactivación económica.
} 
económico de mercado que limitan a priori la potencialidad de las nuevas formas de intervención necesarias para tratar con solvencia los problemas climáticos.

En su estudio, la Profesora Aitana de la Varga Pastor - partiendo de la premisa según la cual las Comunidades Autónomas deben ejercer un rol fundamental para lograr los objetivos de transición energética y para abordar los complejos desafíos de la emergencia climática - se pregunta si sigue siendo aplicable la Ley catalana de cambio climático tras la STC $87 / 2019$ y si su esencial sigue intacta. La respuesta al interrogante es positiva, aunque se observa que la sentencia ha impactado negativamente en el nivel de ambición de la Ley y que se confía en el desarrollo de misma para poder implementar los instrumentos de gran calado que siguen vigentes.

La Profesora Manuela Mora Ruíz ha analizado la normativa andaluza concluyendo que, a pesar de la innegable importancia de Ley 8/2018 - que se sitúa en el vértice de la ordenación administrativa frente a la lucha contra el cambio climático y la transición energética - emergen muchas incertidumbres en la medida en que la Ley precisa de un amplio desarrollo reglamentario y de planificación que está aún por venir y que, por ende, impide una valoración firme sobre el nuevo instrumento legal del que se ha dotado la comunidad autónoma. Asimismo, la autora señala una serie de insuficiencias y déficits que podrían ser revisados tanto por la evolución del marco normativo europeo como por la esperada y más ambiciosa ley estatal.

En relación con esta última, el artículo de la Profesora Ana Pallarès Serrano analiza el proyecto de ley de cambio climático y transición energética, aprobado por el Consejo de Ministros el 19 de mayo de 2020, para conocer, primeramente, cual es el contexto, las características y los objetivos que persigue el texto y, así, poder, con posterioridad, determinar y mostrar las luces y las sombras de su contenido regulatorio que, además de objetivos, tiene una importante dimensión programática que remite a un necesario desarrollo normativo. La autora resalta también que la legislación básica sobre protección del medio ambiente abarca gran parte de las medidas e instrumentos contemplados en el proyecto de ley, pues, será tarea de las Comunidades Autónomas desarrollar parte de esta normativa y establecer normas adicionales de protección.

En conclusión, la acción climática y el diseño de los marcos de transición energética requieren una "actualización" constitucional y de la actividad 
administrativa para hacer frente a la complejidad de la emergencia climática y de las múltiples crisis propias de la nueva era geológica. Este rumbo apunta hacia modelos de gobierno multinivel, no solo por razones democráticas, sino también para promover la experimentación e innovación jurídica que tiene, en palabras de Noguiera López, en los "mesoniveles" autonómicos y locales su dimensión más adecuada para ser ensayados y posteriormente implantados en otros ámbitos.

Se trata de la lección sobre los poderes autonómicos y locales como laboratorios de democracia y experimentalismo que enseñaba desde hace tiempo con clarividencia también el Profesor Manuel Ballbé3 ${ }^{39}$, quien hubiera abogado por la necesidad del "efecto California" 40 , como resultado de las regulaciones autonómicas más vanguardistas, y apostado por un autentico federalismo climático innovador.

39 Manuel Ballbé, "El futuro del Derecho administrativo en la globalización: entre la americanización y la europeización", Revista de Administración Pública, núm. 174, 2007, p. 259 ${ }^{40}$ Ibid., pp. 258-259 